\title{
Analysis of Oxford medial unicompartmental knee replacement using the minimally invasive technique in patients aged 60 and above: an independent prospective series
}

\author{
Nanne P. Kort · Jos J. A. M. van Raay • \\ John Cheung · Casper Jolink · Robbie Deutman
}

Received: 14 March 2007/ Accepted: 6 July 2007/Published online: 8 August 2007

(C) Springer-Verlag 2007

\begin{abstract}
We present the outcome of an independent prospective series of phase-3 Oxford medial mobilebearing unicompartmental knee replacement surgery. Eight surgeons performed the 154 procedures in a communitybased hospital between 1998 and 2003 for patients aged 60 and above. Seventeen knees were revised; in 14 cases a total knee replacement was performed, in 3 cases a component of the unicompartmental knee prosthesis was revised, resulting in a survival rate of $89 \%$ during these 2-7 years follow-up interval. This study shows that mobile-bearing unicompartmental knee replacement using a minimally invasive technique is a demanding procedure. The study emphasises the importance of routine in surgical management and strict adherence to indications and operation technique used to reduce outcome failure.
\end{abstract}

Keywords Knee - Unicompartmental · Replacement · Mobile bearing

\section{Introduction}

Modifications over the past 15 years have improved unicompartmental knee replacement surgery, as indicated in

The authors of this manuscript have no financial relationships to disclose with companies mentioned in this article.

\section{N. P. Kort ( $\square)$}

Department of Orthopaedics, Maasland Hospital,

Sittard, The Netherlands

e-mail: n.kort@orbisconcern.nl

J. J. A. M. van Raay · J. Cheung · C. Jolink - R. Deutman Department of Orthopaedics, Martini Hospital,

Groningen, The Netherlands recent reports on the procedure [1-4]. The designers [5] (the originators) of the Oxford unicompartmental knee prosthesis (Biomet, Warsaw, IN) reported in 1998 a $97.7 \%$ cumulative survival rate of 10 years. An independent series with a 15-year survival analysis claimed a $94 \%$ cumulative survival rate [6]. The outcome was dependent on proper patient selection, surgical techniques and implant design, $[4,7]$ and the results have been attributed to improvements in these factors. The procedure is now performed through a short incision from the medial pole of the patella to the tibial tuberosity. Using this approach, there is little damage to the extensor mechanism, the patella is not dislocated, and the suprapatellar synovial pouch remains intact. As a result, patients recover more quickly. Patients achieve knee flexion, straight leg-raising, and independent stair-climbing three times faster than after total knee replacement (TKR) and twice faster than after open unicompartmental knee replacement surgery [8]. The minimally invasive procedure has been shown to be reliable and effective [9].

Because of the favourable published clinical results, surgeons at the Martini Hospital in Groningen, the Netherlands, began using the Oxford knee prosthesis in 1998. The goal of this independent prospective study for patients 60 years of age and above was to compare and evaluate the clinical midterm results of the Oxford phase-3 unicompartmental knee replacement using the minimally invasive technique in a community hospital.

\section{Materials and methods}

Between December 1998 and 2003, 154 successive Oxford unicompartmental knee replacements were performed in patients 60 years of age and above (Table 1). Of these, 132 patients underwent unilateral surgery, 10 patients 
Table 1 Oxford phase-3 unicompartmental knee replacement

\begin{tabular}{ll}
\hline Criteria & Results \\
\hline Number of patients & 132 \\
Number of knees & 154 \\
Left/right knee (\%) & $53.8 / 46.2$ \\
Age (mean/range, in years) & $69.2(60-93)$ \\
Gender (M/W) & $57(40 \%) / 86(60 \%)$ \\
BMI & $30.7 \pm 4.9$ \\
Follow-up range & $2-7$ years \\
\hline
\end{tabular}

underwent bilateral surgery on separate occasions, and 1 patient underwent concomitant bilateral surgery in the same OR session. There were 86 women; the average patient's age was 69.2 years (range 60-93 years). All patients gave informed consent before their inclusion in this prospective study. Five patients had secondary osteoarthritis because of previous trauma. The remaining patients had primary osteoarthritis.

Standardised anteroposterior radiographs were obtained with the patient in a weight-bearing position (standing), and lateral radiographs were obtained with the patient in a non-weight-bearing position (the patient lying horizontally). The radiographs were examined for loosening or radiolucency around the femoral and tibial components, and the anatomical axis of the limb was measured. The imaging criterion for no increased risk for loosening of the bone was a $<2 \mathrm{~mm}$ thick radiolucent line [10]. The presence of osteoarthritic changes in the nonreplaced compartment was graded according to the Ahlback classification of osteoarthritis (Table 2) [11]. These procedures were performed by eight senior staff surgeons over the study period. Mean preoperative range of motion was $122.9 \pm 8.9^{\circ}$ of flexion and $-0.7 \pm 4.5^{\circ}$ of extension.

The results (preoperative, intraoperative, and follow-ups at 3 months, 6 months, and 1 year) were prospectively recorded with a historical record, procedure record, Knee Society score, SF-36 questionnaire (short form consisting of 36 questions), and the Western Ontario McMaster (WOMAC) score. Knee Society score ratings of excellent (90-100 points) and good (80-89 points) indicated success. The preoperative scores of the patients are presented in Table 3.

Table 2 The Alhback radiological scoring system for estimating the severity of $\mathrm{OA}$

\begin{tabular}{ll}
\hline Grade 0 & Normal \\
Grade 1 & Joint narrowing \\
Grade 2 & Joint obliteration \\
Grade 3 & Bone destruction $<5 \mathrm{~mm}$ \\
Grade 4 & Bone destruction $>5 \mathrm{~mm}$ \\
Grade 5 & Subluxation \\
\hline
\end{tabular}

Preoperative weight-bearing radiographs showed that the knees had an average femorotibial alignment of $2.4^{\circ}$ of valgus (range $8^{\circ}-3^{\circ}$ of varus). Thirty-seven knees had grade-1 Ahlback osteoarthritis [11] in the lateral compartment on the preoperative radiographs, and one had

Table 3 Scoring results of the non-revised patients

\begin{tabular}{|c|c|}
\hline Scoring & Results \\
\hline \multicolumn{2}{|c|}{ Knee society score } \\
\hline \multicolumn{2}{|l|}{ Knee score } \\
\hline Preoperative & 39.2 (SD 18.2) \\
\hline Postoperative & 89.4 (SD 14.0) \\
\hline \multicolumn{2}{|l|}{ Function } \\
\hline Preoperative & $55.8(\mathrm{SD} \mathrm{14.3)}$ \\
\hline Postoperative & 77.1 (SD 24.7) \\
\hline \multicolumn{2}{|l|}{ Total score } \\
\hline Preoperative & $47.6(\mathrm{SD} 12.3)$ \\
\hline Postoperative & 83.4 (SD 16.8) \\
\hline \multicolumn{2}{|l|}{ WOMAC score } \\
\hline \multicolumn{2}{|l|}{ Pain } \\
\hline Preoperative & 50.3 (SD 18.7) \\
\hline Postoperative & 78.6 (SD 21.5) \\
\hline \multicolumn{2}{|l|}{ Stiffness } \\
\hline Preoperative & $51.2($ SD 22.6) \\
\hline Postoperative & 71.2 (SD 20.8) \\
\hline \multicolumn{2}{|l|}{ Function } \\
\hline Preoperative & $50.6($ SD 20.7) \\
\hline Postoperative & 76.2 (SD 20.4) \\
\hline \multicolumn{2}{|c|}{ SF-36 questionnaire } \\
\hline \multicolumn{2}{|l|}{ Function } \\
\hline Preoperative & 35.7 (SD 17.6) \\
\hline Postoperative & $56.1(\mathrm{SD} 24.5)$ \\
\hline \multicolumn{2}{|l|}{ Physical } \\
\hline Preoperative & $28.2($ SD 37.2) \\
\hline Postoperative & $57.2(\mathrm{SD} \mathrm{44.3)}$ \\
\hline \multicolumn{2}{|l|}{ Pain } \\
\hline Preoperative & $32.7(\mathrm{SD} 19.2)$ \\
\hline Postoperative & $59.8($ SD 26.5) \\
\hline \multicolumn{2}{|l|}{ Health } \\
\hline Preoperative & 63.7 (SD 22.2) \\
\hline Postoperative & $61.4(\mathrm{SD} 21.7)$ \\
\hline \multicolumn{2}{|l|}{ Social function } \\
\hline Preoperative & $52.6(\mathrm{SD} 17.1)$ \\
\hline Postoperative & 64.5 (SD 17.6) \\
\hline \multicolumn{2}{|l|}{ Emotional } \\
\hline Preoperative & 64.5 (SD 44.6) \\
\hline Postoperative & 70.5 (SD 40.7) \\
\hline \multicolumn{2}{|l|}{ Mental health } \\
\hline Preoperative & 73.7 (SD 17.9) \\
\hline Postoperative & 75.1 (SD 18.8) \\
\hline
\end{tabular}

$S D$ standard deviation 
grade-2 Ahlback osteoarthritis. The preoperative skyline view of the patellofemoral joint showed no bone loss with eburnation and longitudinal grooving in all the cases.

All medial compartment arthroplasties were performed using the minimally invasive technique and under tourniquet control. The discharge criteria were control of immediate postoperative pain and the ability to flex the operated knee to a minimum of $90^{\circ}$ with no lack of extension. All complications and revisions were reported, and a revision was defined as any surgical procedure resulting in removal or exchange of any of the prosthetic components.

\section{Results}

At the time of follow-up, two patients who had no known revisions were lost for the follow-up. The remaining 130 patients were available for follow-up. At the final followup, June 2006, revision TKR was performed in 14 knees and a prosthetic component was exchanged in three knees. An overview of the revisions is given in Table 4.

One bearing was replaced because of luxation after a hyperflexion trauma. A new bearing of the same size was inserted, and no recurrence of luxation was seen at followup. In another case of luxation of the bearing, the femoral component, and the bearing were changed 9 months after the primary surgery. The fixation of the femoral component in this case was insufficient. The multiple small drill holes were not made, and there was no cement in the large drill hole. With flexion, the loose femoral component moved distally, causing luxation of the bearing. The tibial component and bearing revision was performed seven months after the primary surgery because of misalignment of this tibial component. With flexion, there was impingement of the bearing with the tibial component, causing a clicking sensation and rotation of the bearing.

Table 4 Revisions of Oxford phase-3 knee replacement surgery

\begin{tabular}{ll}
\hline & Incidence \\
\hline Revision of a component of UKA & 3 \\
Revision of the mobile bearing & 1 \\
Revision of the femoral component and the bearing & 1 \\
Revision of the tibial component and the bearing & 1 \\
Conversion to a TKR & 14 \\
Reason for revision to a TKA & \\
Inappropriate indication & 1 \\
Misalignment and loosening & 5 \\
Infection & 1 \\
Progression of osteoarthritis in lateral compartment & 4 \\
Persisting anteromedial pain >1 year & 3 \\
\hline
\end{tabular}

In one case, there was grade-2 Ahlback osteoarthritis [11] in the lateral compartment on the preoperative radiograph. This patient had no relief of preoperative pain, and the knee underwent TKR 18 months after the primary surgery. In five cases loosening of the components occurred; misalignment of the components is probably caused by impingement of the bearing. One patient had a deep Staphylococcus aureus infection, and a two-stage procedure was performed leading to a TKR. In four cases of revision, progression of osteoarthritis was seen in the lateral compartment with reported pain on the lateral side. These patients had a mean postoperative anatomical axis, femorotibial alignment of $18.6^{\circ}$. This overcorrection causes overloading of the lateral compartment with progression of arthritis in that compartment.

Three patients with persisting anteromedial pain underwent revision. In two cases, no cause was found, and in both pain persisted after TKR. In the third case, the synovial biopsy showed synovitis villonodularis pigmentosa, and after the TKR this patient was pain-free. Except for the two patients with persisting anteromedial pain, all patients with a conversion to TKA were pain-free. No special augmentations or revision prosthetic components were necessary in these procedures; there were no bone defects that required the use of particulate autograft or allograft, and primary cruciate-retaining TKA was used in the revisions.

Postoperative complications occurred after the primary unicompartmental knee replacements. One patient had a traumatic medial tibia plateau fracture 4 weeks postoperatively, which was treated conservatively. Another patient developed hemarthrosis that required extended hospitalisation; this was resolved with conservative treatment. There was one deep infection, and no deep venous thrombosis was reported.

At the time of the most recent follow-up, average flexion was $125.8 \pm 13.8^{\circ}$, with two patients achieving $<90^{\circ}$ flexion. The average flexion deformity/extension was $0.3 \pm 2.2^{\circ}$.

The postoperative scores of those patients who did not undergo revision (140 knees) at the latest follow-up are presented in Table 3. The Knee Society score total was 83.4. All three WOMAC scores improved. For the SF-36, the function, physical, and pain scores showed an improvement in the outcome; the other scores remained approximately the same.

The final follow-up radiographs showed an average anatomical axis, femorotibial alignment of $8.8^{\circ}$ of valgus (range $4^{\circ}-22^{\circ}$ of valgus). The knees were corrected by an average of $6.4^{\circ}$ (range $2^{\circ}-14^{\circ}$ ). This relative overcorrection gives increased stress on the lateral compartment. Signs of osteoarthritis progression in the uninvolved tibiofemoral compartment on the radiograph at the last follow-up were 
noted in 43 knees (grade-1 Ahlback osteoarthritis in 39 knees and grade-2 Ahlback osteoarthritis in four knees). No grade- 3 or -4 changes were noted. At final radiographic evaluation, no component showed evidence of loosening. No knees had $>2 \mathrm{~mm}$ of tibial cement-bone radiolucency. There were no radiolucent lines seen at the posterior aspect of the femoral components.

Seventeen knees were revised, resulting in a survival rate of $89 \%$ in these $2-7$ years of follow-up interval.

\section{Discussion}

The purpose of this prospective study was to evaluate midterm durability of Oxford unicompartmental knee replacement surgery for patients 60 years of age and older. We acknowledge that the present study has the limitations of a midterm follow-up. However, longer follow-up for this phase-III version with the minimally invasive technique is not possible, because the current version has been available only since 1998 [7]. Besides, most technical failures occur within the first 2 years [12].

In these 2-7 years of follow-up interval, $11 \%$ of unicompartmental knee arthroplasties in all patients needed revision-a survival rate of $89 \%$. These results are considerably lower compared to the designer [5] series or the independent series [6].

The primary need for revision surgery could be attributed to indication and technical failures. Thirteen of the 17 revisions were probably related to human error, the remaining four are in one case a hyperflexion trauma and luxation of the bearing, one case with deep infection, and two cases with unexplained persisting anteromedial pain. Misalignment of the components was the primary cause of technical failure. With the minimally invasive technique, the visual field is restricted, making mobile-bearing unicompartmental knee replacement surgery a demanding procedure. Introduction of the minimally invasive option makes the terms surgical technique and pitfalls actual again.

For the remaining 113 patients (140 knees) who did not undergo revision, the Knee Society score, WOMAC and SF-36 questionnaires showed an improvement in the outcome. All three scores indicated less pain and improvement in function, as confirmed by an average clinical average flexion of $126^{\circ}$ at the latest follow-up. The Knee Society score total of 83.4 indicates a successful outcome.

Over the 7-year period of our study, eight senior surgeons performed the operation with an average of $<10$ procedures a year per surgeon. All surgeons attended the instructional course organized by the designer group. There is no evidence for a learning curve in our study. The outcome should be attributed to the number of operations performed. As a result of the relatively low survival rate of this study, the number of senior surgeons performing the procedure in this hospital is now reduced to two.

\section{Conclusion}

Careful patient selection, surgeon experience, and proper instrumentation and surgical technique are important factors in mobile-bearing unicompartmental knee replacement surgery [13, 14]. For unicompartmental replacement surgery, long-term results are related to the number performed by the unit [14]. The surgeon should be well versed in the routine, indications, and technique of this procedure to minimise failure rates.

\section{References}

1. Verdonk R, Cottenie D, Almqvist KF, Vorlat P (2005) The Oxford unicompartmental knee prosthesis: a 2-14 year followup. Knee Surg Sports Traumatol Arthrosc 13:163-166

2. Tabor OB Jr, Tabor OB, Bernard M, Wan JY (2005) Unicompartmental knee arthroplasty: long-term success in middle-age and obese patients. J Surg Orthop Adv 14:59-63

3. Saxler G, Temmen D, Bontemps G (2004) Medium-term results of the AMC-unicompartmental knee arthroplasty. Knee 11:349355

4. Price AJ, Waite JC, Svard U (2005) Long-term clinical results of the medial Oxford unicompartmental knee arthroplasty. Clin Orthop Relat Res 435:171-180

5. Murray DW, Goodfellow JW, O'Connor JJ (1998) The Oxford medial unicompartmental arthroplasty: a ten-year survival study. J Bone Joint Surg Br 80:983-939

6. Svard UC, Price AJ (2001) Oxford medial unicompartmental knee arthroplasty. A survival analysis of an independent series. J Bone Joint Surg Br 83:191-194

7. Murray DW (2005) Mobile bearing unicompartmental knee replacement. Orthopedics 28:985-987

8. Price AJ, Webb J, Topf H, Dodd CA, Goodfellow JW, Murray DW (2001) Rapid recovery after oxford unicompartmental arthroplasty through a short incision. J Arthroplasty 16:970-976

9. Pandit H, Jenkins C, Barker K, Dodd CA, Murray DW (2006) The Oxford medial unicompartmental knee replacement using a minimally-invasive approach. J Bone Joint Surg Br 88:54-60

10. Tibrewal SB, Grant KA, Goodfellow JW (1984) The radiolucent line beneath the tibial components of the Oxford meniscal knee. J Bone Joint Surg Br 66:523-528

11. Ahlback S (1968) Osteoarthrosis of the knee. A radiographic investigation. Acta Radiol Diagn (Stockh) Suppl 277:7-72

12. Lewold S, Goodman S, Knutson K, Robertsson O, Lidgren L (1995) Oxford meniscal bearing knee versus the Marmor knee in unicompartmental arthroplasty for arthrosis. A Swedish multicenter survival study. J Arthroplasty 10:722-731

13. Jeer PJ, Keene GC, Gill P (2004) Unicompartmental knee arthroplasty: an intermediate report of survivorship after the introduction of a new system with analysis of failures. Knee 11:369-374

14. Robertsson O, Knutson K, Lewold S, Lidgren L (2001) The routine of surgical management reduces failure after unicompartmental knee arthroplasty. J Bone Joint Surg Br 83:45-49 